\title{
Synthesis and Diels-Alder cycloaddition reaction of norbornadiene and benzonorbornadiene dimers
}

\author{
Bilal Nişancı, Erdin Dalkılıç, Murat Güney and Arif Daştan*
}

\section{Full Research Paper}

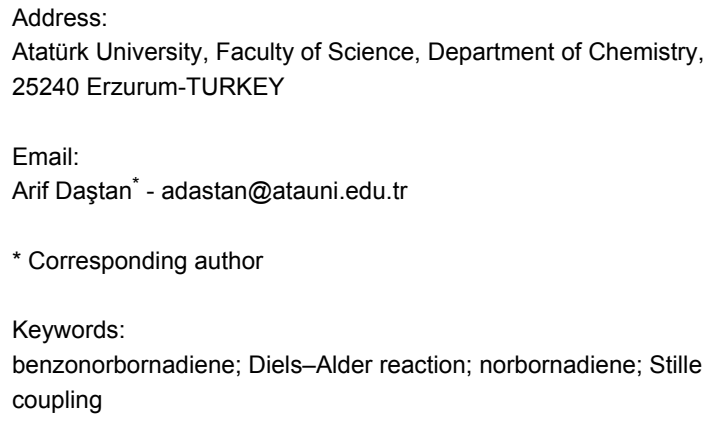

Beilstein Journal of Organic Chemistry 2009, 5, No. 39 doi:10.3762/bjoc.5.39

Received: 12 June 2009

Accepted: 07 August 2009

Published: 11 August 2009

Editor-in-Chief: J. Clayden

(c) 2009 Nişancı et al; licensee Beilstein-Institut.

License and terms: see end of document.

\begin{abstract}
Dimeric forms of norbornadiene and benzonorbornadiene were synthesized starting with known monobromide derivatives. The Diels-Alder cycloaddition reaction of dimers with TCNE and PTAD was investigated and new norbornenoid polycyclics were obtained. All compounds were characterized properly using NMR spectroscopy.
\end{abstract}

\section{Introduction}

Norbornadiene (1) and related compounds are of great scientific interest because of their unusual geometry and high reactivity. For example, these compounds exhibit a unique behavior in the cationic Wagner-Meerwein rearrangement [1-10], in the solvolytic reactivity [11], in the photochemical di- $\pi$-methane rearrangement [12-15], as well as in other instances [16-22]. Therefore, functionalizations of these compounds are important. In this study, we investigated the synthesis and Diels-Alder cycloaddition reaction of norbornadiene and benzonorbornadiene dimers.

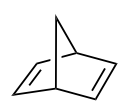

1

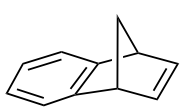

2

\section{Results and Discussion}

One of the starting materials, 2-bromobenzonorbornadiene 4 was synthesized using a procedure described in the literature [15,23] (Scheme 1). Photochemical bromination of benzonorbornadiene 2 with 1,2-dibromotetrachloroethane gave isomeric dibromides 3 in high yield. Dehydrobromination reaction of dibromides 3 with potassium tert-butoxide resulted in the formation of monobromide $\mathbf{4}$. The other starting material $\mathbf{5}$ was obtained using the reported procedures based on the use of potassium tert-butoxide/n-butyllithium super-base by starting with commercially available norbornadiene [24-27].

When 2-bromobenzonorbornadiene 4 was treated with $n$-BuLi at $-78{ }^{\circ} \mathrm{C}$ and the resulting anion was quenched with trimethyltin chloride, a single trimethyltin derivative 6 was 

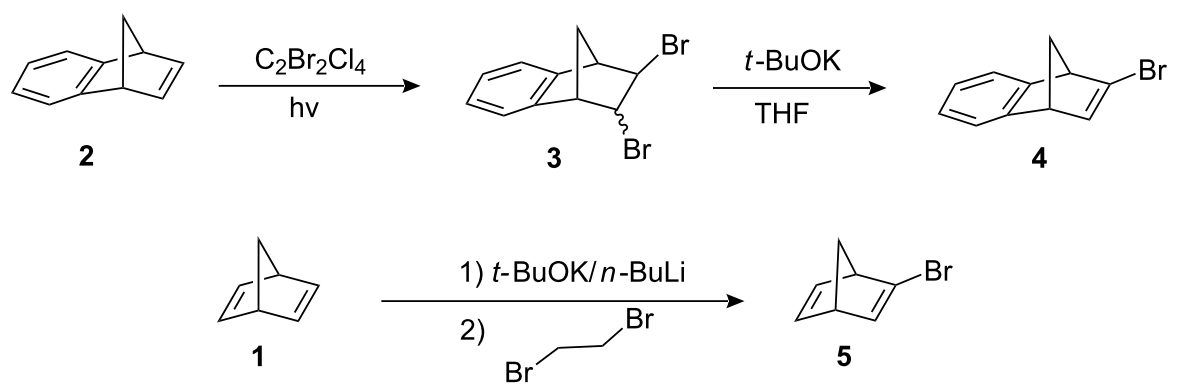

Scheme 1: Synthesis of starting materials 4 and 5.

observed in the crude reaction mixture and was finally isolated in $91 \%$ yield. Copper salts have been successfully employed for Stille-type hetero-coupling between unsaturated halides and stannanes [28,29]. Treatment of 6 with $\mathrm{Cu}\left(\mathrm{NO}_{3}\right)_{2} \cdot 3 \mathrm{H}_{2} \mathrm{O}$ in dry THF at r.t. afforded the first synthesis of the expected dimers 7 and $\mathbf{8}$ in $25 \%$ yield in a $3: 4$ ratio, respectively, besides benzonorbornadiene 2 after column chromatography. The Diels-Alder cycloaddition of dimers 7 and 8 with PTAD (9) and TCNE (10) resulted in the formation of the corresponding products 11-14 in high yields (Scheme 2).

Similarly, tin compound $\mathbf{1 5}$ was synthesized by the reaction of monobromide $\mathbf{5}$ with $n$-BuLi followed by reaction with trimethyltin chloride. Reaction of $\mathbf{1 5}$ with $\mathrm{Cu}\left(\mathrm{NO}_{3}\right)_{2} \cdot 3 \mathrm{H}_{2} \mathrm{O}$ resulted in the formation of dimers 16 and 17 [30]. This reaction offered an alternative synthetic route to norbornadiene dimers 16 and $\mathbf{1 7}$. The isomers $\mathbf{1 6}$ and $\mathbf{1 7}$ could not be separated, but after cycloaddition reaction of the mixture, the corresponding addition products $\mathbf{1 8}-\mathbf{2 1}$ were isolated by chromatographic methods (Scheme 3).

\section{Structural Analyses}

The determination of the structures of dimers 7, 8 and dimers 16, 17 by spectroscopic methods was not simple because the $C_{\mathrm{s}}$ symmetry of the syn dimers and the $C_{2}$ symmetry of the anti dimers and the free rotation around the central $\sigma$ bond make

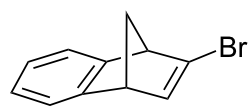

4<smiles>Cc1ccccc1C</smiles><smiles>N#CC(C#N)=C(C#N)C#N</smiles>

10

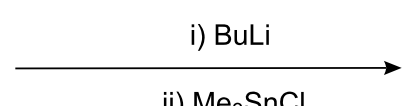

ii) $\mathrm{Me}_{3} \mathrm{SnCl}$

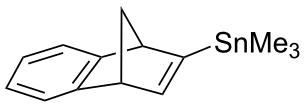

6

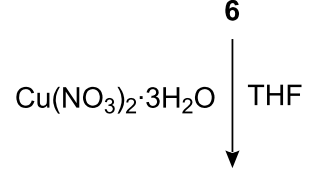

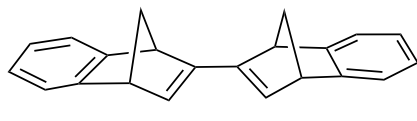

7

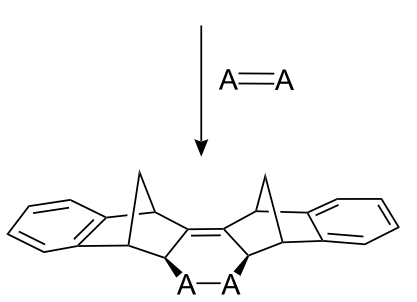

11,12

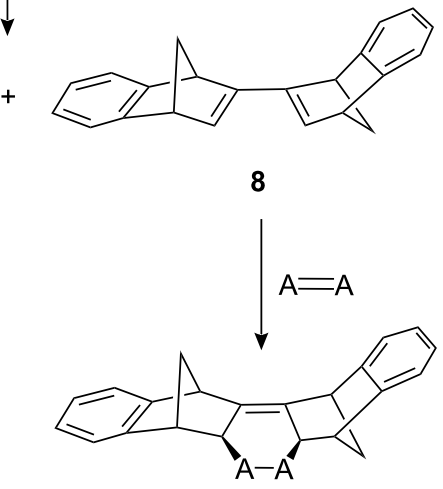

13,14 

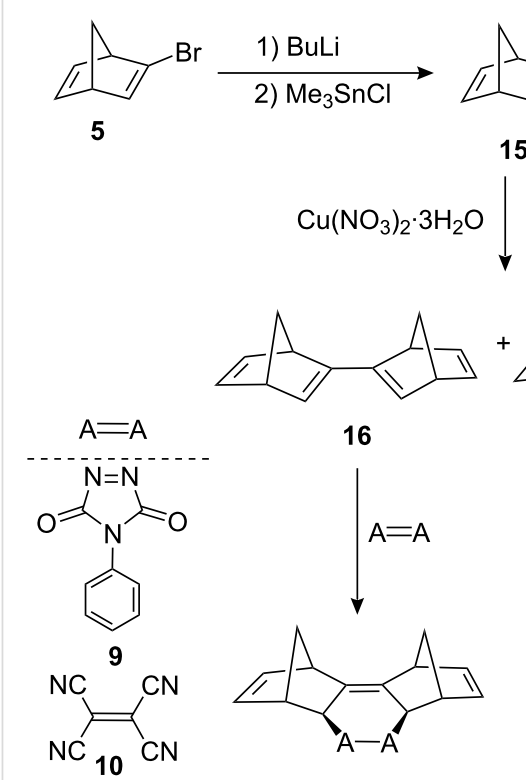

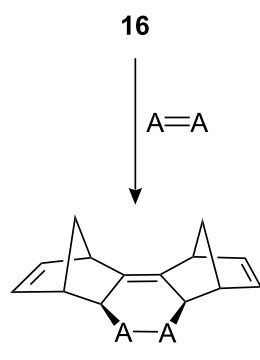

18,19

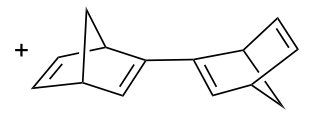

17

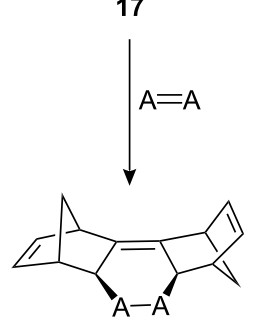

20,21
Scheme 3: Synthesis and Diels-Alder cycloaddition reactions of dimers 16 and 17.

them indistinguishable. To determine which is which, cycloaddition reactions of dimers are more informative. Dimers 7 and 16 give symmetric addition products 11,12 and 18,19 , whereas the reaction of dimers $\mathbf{8}$ and $\mathbf{1 7}$ resulted in the formation of unsymmetrical products 13, 14 and 20, 21 .

For the symmetric addition products $\mathbf{1 1}, \mathbf{1 2}, \mathbf{1 8}$ and 19, there are two possibilities: exo adduct or endo adduct (Figure 1). The coupling constants between the relevant protons in the norbornene unit are very informative to assign the correct configuration of the substituents $[9,10]$. The high value of $J_{34}$ and $J_{3^{\prime} 4^{\prime}}(2.5-3.5 \mathrm{~Hz})$ in the Diels-Alder addition products is uniquely accommodated by the exo orientation of the protons (endo orientation of -A-A- ring) at $\mathrm{C}^{3}$ and $\mathrm{C}^{3^{\prime}}$ carbon atoms. For example, though there is coupling between the protons $\mathrm{H}^{3}$ and $\mathrm{H}^{4}$, there is no measurable coupling between the protons $\mathrm{H}_{3^{\prime}}$ and $\mathrm{H}_{4}$ in anti structures (Figure 1). On the other hand, the absence of any coupling between the related protons confirms the endo orientation of protons at $\mathrm{C}^{3}$ and $\mathrm{C}^{3^{\prime}}$, which in turn proves the exo-orientation of the rings in adduct 11, 12, 18 and 19. The coupling between the protons $\mathrm{H}^{3}\left(\mathrm{H}^{3^{\prime}}\right)$ and $\mathrm{H}^{7 \mathrm{syn}}$ $\left(\mathrm{H}^{7^{\prime} \mathrm{syn}}\right)$ ( $\mathrm{M}$ or $\mathrm{W}$ orientation) also confirms the exo structures for 11, 12, 18 and 19 (Figure 1).

In summary, the synthesis and cycloaddition reaction of norbornadiene and benzonorbornadiene dimers was investigated and new norbornanoid polycyclic compounds, which open up several synthetic and mechanical investigations, were obtained.

\section{Experimental}

General: Melting points are uncorrected. Infrared spectra were obtained from solution in $0.1 \mathrm{~mm}$ cells or $\mathrm{KBr}$ pellets on a regular instrument. The ${ }^{1} \mathrm{H}$ and ${ }^{13} \mathrm{C}$ NMR spectra were recorded on 400 (100) and 200 (50) MHz spectrometers. Apparent splitting is given in all cases. Column chromatography was performed on silica gel (60-mesh, Merck) TLC was carried out on Merck $0.2 \mathrm{~mm}$ silica gel $60 \mathrm{~F}_{254}$ analytical aluminum plates. All substances reported in this paper are meso-compounds or racemates.

Synthesis of (1,4-dihydro-1,4-methano-naphthalen-2yl)trimethylstannane (6): A solution of $n$-BuLi in $n$-hexane (2.7 M, $3.41 \mathrm{~mL}, 9.19 \mathrm{mmol}$ ) was added dropwise to a solution of monobromobenzonorbornadiene 4 (2.03 g, $9.19 \mathrm{mmol})$ in dry THF $(20 \mathrm{~mL})$ at $-78{ }^{\circ} \mathrm{C}$ and the resulting mixture was stirred for $40 \mathrm{~min}$. Trimethyltin chloride $(1.83 \mathrm{~g}, 9.19 \mathrm{mmol})$ was added portionwise and then left to warm to room temperature. The mixture was stirred overnight at room temperature. The crude product was washed with water $(15 \mathrm{~mL})$ and extracted with $\mathrm{Et}_{2} \mathrm{O}(2 \times 50 \mathrm{~mL})$ and then the combined ethereal extracts were dried over $\mathrm{MgSO}_{4}$ and concentrated in vacuo. (1,4dihydro-1,4-methano-naphthalen-2-yl)trimethylstannane (6) was obtained as yellow liquid (2.55 g, 91\%). ${ }^{1} \mathrm{H}$ NMR (400 $\mathrm{MHz}, \mathrm{CDCl}_{3}$ ): $\delta 7.26-7.20\left(\mathrm{~m}, 2 \mathrm{H}\right.$, aryl), $7.06\left(\mathrm{~d}, J_{3,4}=2.9 \mathrm{~Hz}\right.$, $\left.1 \mathrm{H}, \mathrm{H}_{3}\right), 6.98-6.94(\mathrm{~m}, 2 \mathrm{H}$, aryl $), 4.08\left(\mathrm{~m}, 1 \mathrm{H}, \mathrm{H}_{4}\right), 3.98(\mathrm{~m}$, $\left.1 \mathrm{H}, \mathrm{H}_{1}\right), 2.24\left(\mathrm{~m}, 2 \mathrm{H}, \mathrm{H}_{9 \text { syn }}\right.$ and $\left.\mathrm{H}_{9 a n t i}\right), 0.18\left(\mathrm{~s}, 9 \mathrm{H}, 3 \times \mathrm{CH}_{3}\right)$. ${ }^{13} \mathrm{C}$ NMR $\left(100 \mathrm{MHz}, \mathrm{CDCl}_{3}\right): \delta 155.61,153.22,151.95$, 151.92, 124.36, 124.18, 121.62, 121.58, 69.65, 55,77, 52.05, -9.70 .

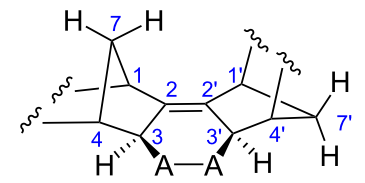

anti

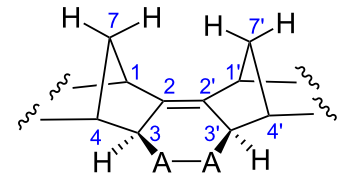

syn-exo

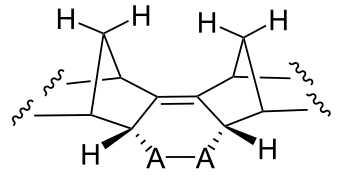

syn-endo 
Reaction of (1,4-dihydro-1,4-methano-naphthalen-2yl)trimethylstannane (6) with $\mathbf{C u}\left(\mathrm{NO}_{3}\right)_{2} \cdot \mathbf{3 H}_{2} \mathrm{O}$ : $\mathrm{Copper}(\mathrm{II})$ nitrate trihydrate $(345 \mathrm{mg}, 1.4 \mathrm{mmol})$ was added portionwise to a solution of $6(435 \mathrm{mg}, 1.4 \mathrm{mmol})$ in THF $(6 \mathrm{~mL})$ at room temperature. The blue solution turned green within $1 \mathrm{~h}$. The crude reaction mixture was diluted with $\mathrm{Et}_{2} \mathrm{O}(100 \mathrm{~mL})$ and then washed with $5 \% \mathrm{NH}_{3}(15 \mathrm{~mL})$. The organic phase was dried over $\mathrm{MgSO}_{4}$ and concentrated in vacuo. The residue was chromatographed on neutral aluminum oxide (150 g) eluted with hexane. The first fraction was benzonorbornadiene (155 $\mathrm{mg}, 57 \%)$. The second fraction was anti isomer $8(28 \mathrm{mg}, 14 \%)$. ${ }^{1} \mathrm{H} \mathrm{NMR}\left(400 \mathrm{MHz}, \mathrm{CDCl}_{3}\right): \delta 7.15-6.79\left(\mathrm{~m}, 8 \mathrm{H}, \mathrm{H}^{\text {aryl }}\right), 6.56$ $\left(\mathrm{d}, J_{3,4}=J_{3^{\prime}, 4^{\prime}}=2.9 \mathrm{~Hz}, 2 \mathrm{H}, \mathrm{H}^{3}\right.$ and $\left.\mathrm{H}^{3^{\prime}}\right), 3.92\left(\mathrm{~m}, 2 \mathrm{H}, \mathrm{H}^{4}\right.$ and $\left.\mathrm{H}^{4^{\prime}}\right), 3.86\left(\mathrm{~m}, 2 \mathrm{H}, \mathrm{H}^{1}\right.$ and $\left.\mathrm{H}^{1^{\prime}}\right), 2.40(\mathrm{dt}$, A Part of AB system, $J_{9 \text { syn }, 9 \text { anti }}=J_{9^{\prime}{\text { syn }, 9^{\prime} \text { anti }}}=7.1 \mathrm{~Hz}, J_{9 \text { syn }, 1}=J_{9 \text { syn }, 4}=J_{9^{\prime} \text { syn }, 1^{\prime}}=$ $J_{9^{\prime} s y n, 4^{\prime}}=1.5 \mathrm{~Hz}, 2 \mathrm{H}, \mathrm{H}^{9 s y n}$ and $\left.\mathrm{H}^{9 ' s y n}\right), 2.25$ (bd, B part of $\mathrm{AB}$ system, $\left.J_{9 a n t i, 9 s y n}=J_{9^{\prime} a n t i, 9^{\prime}{ }^{\prime} s y}=7.1 \mathrm{~Hz}, 2 \mathrm{H}, \mathrm{H}^{9 a n t i, 9^{\prime} a n t i}\right) .{ }^{13} \mathrm{C}$ NMR $\left(100 \mathrm{MHz}, \mathrm{CDCl}_{3}\right): \delta 151.89,151.46,150.65,133.96$, $124.40,124.32,121.67,120.97,68.76,52.12,50.89$. The third fraction was the $s y n$-dimer $7(23 \mathrm{mg}, 11 \%)$. Colorless crystals from $\mathrm{CH}_{2} \mathrm{Cl}_{2} / n$-hexane (1:3). mp 152-154 ${ }^{\circ} \mathrm{C} .{ }^{1} \mathrm{H}$ NMR (400 $\left.\mathrm{MHz}, \mathrm{CDCl}_{3}\right): \delta 7.29-6.94\left(\mathrm{~m}, 8 \mathrm{H}, \mathrm{H}^{\text {aryl }}\right), 6.61\left(\mathrm{~d}, J_{3,4}=J_{3^{\prime}, 4^{\prime}}=\right.$ $2.9 \mathrm{~Hz}, 2 \mathrm{H}, \mathrm{H}^{3}$ and $\left.\mathrm{H}^{3^{\prime}}\right), 3.90\left(\mathrm{~m}, 2 \mathrm{H}, \mathrm{H}^{4}\right.$ and $\left.\mathrm{H}^{4^{\prime}}\right), 3.80(\mathrm{~m}, 2 \mathrm{H}$, $\mathrm{H}^{1}$ and $\left.\mathrm{H}^{1^{\prime}}\right), 2.21\left(\mathrm{dt}, \mathrm{A}\right.$ Part of $\mathrm{AB}$ system, $J_{9 s y n, 9 a n t i}=$ $J_{9^{\prime} \text { syn }, 9^{\prime} \text { anti }}=7.3 \mathrm{~Hz}, J_{9_{\text {syn }, 1}}=J_{9 \text { syn }, 4}=J_{9^{\prime} \text { syn }, 1^{\prime}}=J_{9^{\prime} \text { syn }, 4^{\prime}}=1.6$ $\mathrm{Hz}, 2 \mathrm{H}, \mathrm{H}^{9 a n t i}$ and $\mathrm{H}^{9^{\prime} a n t i}$ ), 2.17 (bd, B Part of $\mathrm{AB}$ system, $J_{9 \text { anti }, 9 \text { syn }}=J_{9^{\prime} \text { anti, } 9^{\prime} \text { syn }}=7.3 \mathrm{~Hz}, 2 \mathrm{H}, \mathrm{H}^{9 a n t i}$ and $\left.\mathrm{H}^{9^{\prime} a n t i}\right) .{ }^{13} \mathrm{C}$ NMR $\left(100 \mathrm{MHz}, \mathrm{CDCl}_{3}\right): \delta 151.88,151.55,151.32,134.38$, $124.59,124.41,121.70,121.15,67.71,51.59,50.72$. IR (KBr, $\left.\mathrm{cm}^{-1}\right)$ : 3067, 2981, 2936, 2866, 1455, 1317, 1270, 1226, 1199, 1149, 1068, 1011, 909, 750, 735. MS (70 eV) m/z: $282.5\left(\mathrm{M}^{+}\right.$, 32), 267.5 (21), 239.4 (5), 202.4 (2), 178.4 (5), 167.3 (26), 165.3 (32), 141.2 (28), 117.2 (71), 115.2 (56), 89.1 (6), 63.1 (3).

Cycloaddition reaction of the dimer 7 with PTAD (9): A solution of the syn dimer 7 (40 mg, $0.14 \mathrm{mmol})$ and PTAD (25 $\mathrm{mg}, 0.14 \mathrm{mmol}$ ) in $4 \mathrm{~mL}$ of $\mathrm{CH}_{2} \mathrm{Cl}_{2}$ was stirred at room temperature for $30 \mathrm{~min}$. The solvent was removed under reduced pressure. The crude product was purified by crystallization from $\mathrm{CH}_{2} \mathrm{Cl}_{2} / n$-hexane (3:1) to give syn cycloadduct 11 (55 mg, $89 \%$ ). Yellow crystals, mp $182-184{ }^{\circ} \mathrm{C} .{ }^{1} \mathrm{H}$ NMR $(400 \mathrm{MHz}$, $\left.\mathrm{CDCl}_{3}\right): \delta 7.60-7.12(\mathrm{~m}, 13 \mathrm{H}), 4.66(\mathrm{~s}, 2 \mathrm{H}), 4.24(\mathrm{~s}, 2 \mathrm{H}), 3.75$ (d, $J=1.5 \mathrm{~Hz}, 2 \mathrm{H}), 2.21$ (dq, A Part of AB system, $J=9.4 \mathrm{~Hz}$, $J=1.5 \mathrm{~Hz}, 2 \mathrm{H}$ ), 2.14 (dt, B Part of AB system, $J=9.4 \mathrm{~Hz}, J=$ $1.4 \mathrm{~Hz}, 2 \mathrm{H}) .{ }^{13} \mathrm{C} \mathrm{NMR}\left(100 \mathrm{MHz}, \mathrm{CDCl}_{3}\right): \delta 151.74,144.59$, 144.54, 132.02, 131.84, 129.27, 128.28, 127.38, 127.05, 126.04, $123.09,121.94,59.25,48.73,48.20,47.18$. IR $\left(\mathrm{KBr}, \mathrm{cm}^{-1}\right)$ : 3048, 2976, 2941, 1762, 1702, 1600, 1502, 1439, 1419, 1343, 1265, 1140. MS (70 eV) m/z: $458.4\left(\mathrm{M}^{+}, 3\right), 344.0(5), 282.0$ (10), 280.8 (7), 165.6 (24), 119.4 (43), 116.4 (100), 91.3 (43).
Cycloaddition reaction of the dimer 7 with TCNE (10): A solution of the syn dimer $7(50 \mathrm{mg}, 0.17 \mathrm{mmol})$ and TCNE (10, $23 \mathrm{mg}, 0.17 \mathrm{mmol}$ ) in $5 \mathrm{~mL}$ of $\mathrm{CH}_{2} \mathrm{Cl}_{2}$ was stirred at room temperature for overnight. The solvent was removed under reduced pressure. The crude product was purified by crystallization from $\mathrm{CH}_{2} \mathrm{Cl}_{2} / n$-hexane (3:1) to give syn cycloadduct 12 (68 mg, 93\%). White crystals, mp 230-232 ${ }^{\circ} \mathrm{C} .{ }^{1} \mathrm{H}$ NMR (400 $\mathrm{MHz} \mathrm{CDCl}_{3}$ ): $\delta$ 7.54-7.17 (m, 8H, $\left.\mathrm{H}^{\text {aryl }}\right), 4.22(\mathrm{~s}, 2 \mathrm{H}), 3.86$ (m, 2H), 2.48 (bd, A Part of AB system, 2H, $J=10.3 \mathrm{~Hz}$ ), 2.45 (m, 2H), 2.22 (d, B Part of AB system, $2 \mathrm{H}, J=10.3 \mathrm{~Hz}) \cdot{ }^{13} \mathrm{C}$ NMR (100 MHz, $\left.\mathrm{CDCl}_{3}\right): \delta 145.93,144.40,133.14,127.76$, $127.32,122.17,122.06,112.08,110.87,50.01,48.54,47.81$, 46.73, 45.90. IR (KBr, cm $\left.{ }^{-1}\right): 3050,2955,2872,2306,2254$, $2217,1463,1318,1265,1120,1153,1013,981,785,704 . \mathrm{MS}$ (70 eV) $\mathrm{m} / \mathrm{z}: 410.1\left(\mathrm{M}^{+}, 100\right), 394.1$ (10), 370.1 (37), 345.1 (35), 319.1 (27), 295 (27), 267.1 (45), 265.0 (27), 229.0 (17), 205.0 (32), 176.9 (22), 164.9 (4), 152.9 (22), 151.9 (30).

Cycloaddition reaction of the dimer 8 with PTAD (9): A solution of the anti dimer (40 mg, $0.14 \mathrm{mmol}$ ) and PTAD (25 $\mathrm{mg}, 0.14 \mathrm{mmol}$ ) in $4 \mathrm{~mL}$ of $\mathrm{CH}_{2} \mathrm{Cl}_{2}$ was stirred at room temperature for $30 \mathrm{~min}$. The solvent was removed under reduced pressure. The crude product was purified by crystallization from ether $/ n$-hexane (2:1) to give anti cycloadduct 13 (58 mg, 90\%). Yellow crystals, mp $168-170{ }^{\circ} \mathrm{C} .{ }^{1} \mathrm{H} \mathrm{NMR}\left(400 \mathrm{MHz}, \mathrm{CDCl}_{3}\right)$ : $\delta$ 7.57-7.00 (m, 13H, $\left.\mathrm{H}^{\mathrm{aryl}}\right), 4.94(\mathrm{~m}, 1 \mathrm{H}), 4.52(\mathrm{~d}, J=2.3 \mathrm{~Hz}$, 1H), $4.38(\mathrm{~m}, 1 \mathrm{H}), 4.09(\mathrm{~m}, 1 \mathrm{H}), 3.34(\mathrm{~m}, 1 \mathrm{H}), 2.40$ (dt, A part of AB system, $J=7.7 \mathrm{~Hz}, J=1.5 \mathrm{~Hz}, 1 \mathrm{H}), 2.36$ (dt, B part of AB system, $J=7.7 \mathrm{~Hz}, J=1.5 \mathrm{~Hz}, 1 \mathrm{H}), 1.43$ (bd, A part of AB system, $J=10.7 \mathrm{~Hz}, 1 \mathrm{H}), 1.25(\mathrm{~m}, 1 \mathrm{H}), 0.46(\mathrm{bd}, \mathrm{B}$ part of AB system, $J=10.7 \mathrm{~Hz}, 1 \mathrm{H}) .{ }^{13} \mathrm{C} \mathrm{NMR}\left(100 \mathrm{MHz} \mathrm{CDCl}_{3}\right): \delta$ 155.20, 154.38, 150.70, 147.28, 147.18, 145.68, 142.93, 129.50, $129.35,128.80,128.53,127.91,127.53,125.93,125.85,125.62$, $125.41,123.17,122.43,121.92,69.32,63.91,62.90,50.90$, 49.64, 49.53, 49.27, 45.43. IR (KBr, $\left.\mathrm{cm}^{-1}\right)$ : 3065, 2961, 2923, 2851, 1718, 1497, 1412, 1262, 1135, 1091, 1023, 801. MS (70 eV) $m / z$ : $410.1\left(\mathrm{M}^{+}, 100\right), 394.1$ (10), 370.1 (33), 345.1 (31), 319.1 (26), 267.1 (45), 205.0 (33), 164.9 (44), 151.9 (32).

Cycloaddition reaction of the dimer 8 with TCNE (10): A solution of the anti dimer 8 (40 mg, $0.14 \mathrm{mmol})$ and TCNE (10, $18 \mathrm{mg}, 0.14 \mathrm{mmol}$ ) in $5 \mathrm{~mL}$ of $\mathrm{CH}_{2} \mathrm{Cl}_{2}$ was stirred at room temperature for overnight. The solvent was removed under reduced pressure. The crude product was purified by crystallization from $\mathrm{CH}_{2} \mathrm{Cl}_{2} / n$-hexane (3:1) to give anti cycloadduct 14 (53 mg, 91\%). White crystals, mp $240{ }^{\circ} \mathrm{C}$ (dec). ${ }^{1} \mathrm{H}$ NMR (400 $\left.\mathrm{MHz}, \mathrm{CDCl}_{3}\right): \delta 7.55-7.17(\mathrm{~m}, 8 \mathrm{H}), 4.15(\mathrm{~m}, 1 \mathrm{H}), 4.13(\mathrm{~m}$, $1 \mathrm{H}), 3.92(\mathrm{dd}, J=3.5 \mathrm{~Hz}, J=1.5 \mathrm{~Hz}, 1 \mathrm{H}), 3.74(\mathrm{~m}, 1 \mathrm{H}), 3.41$ (dd, $J=3.5 \mathrm{~Hz}, J=1.5 \mathrm{~Hz}, 1 \mathrm{H}), 2.52(\mathrm{~m}, 1 \mathrm{H}), 2.31$ (dt, A part of AB system, $J=9.5 \mathrm{~Hz}, J=1.5 \mathrm{~Hz}, 1 \mathrm{H}$ ), 2.11 (dt, A part of AB system, $J=10.3 \mathrm{~Hz}, J=1.5 \mathrm{~Hz}, 1 \mathrm{H}), 2.06$ (dt, B part of AB 
system, $J=9.5 \mathrm{~Hz}, J=1.5 \mathrm{~Hz}, 1 \mathrm{H}), 2.02$ (bd, B part of AB system, $J=10.3 \mathrm{~Hz}, 1 \mathrm{H}) .{ }^{13} \mathrm{C} \mathrm{NMR}\left(100 \mathrm{MHz}, \mathrm{CDCl}_{3}\right): \delta$ $146.29,145.79,144.63,139.70,132.89,132.55,128.80,127.61$, 127.33, 127.30, 126.60, 122.18, 121.98, 120.73, 112.40, 112.33, 109.23, 108.83, 52.93, 50.29, 49.19, 47.86, 47.71, 47.49, 47.43, 46.79, 45.12, 44.55. IR (KBr, $\left.\mathrm{cm}^{-1}\right): 3049,2989,2956,2923$, $2851,2241,1906,1459,1366,1262,1012,984$. MS $(70 \mathrm{eV}) \mathrm{m} /$ $z: 410.1\left(\mathrm{M}^{+}, 40\right), 345.1$ (13), 295.1 (10), 252.0 (7), 205.0 (13), 164.9 (12), 127.9 (8), 117.0 (30), 114.9 (100).

Synthesis of (bicyclo[2.2.1] hepta-2,5-dien-2-yl)trimethylstannane (15): A solution of $n$-BuLi in $n$-hexane $(2.5 \mathrm{M}, 1.2$ $\mathrm{ml}, 2.9 \mathrm{mmol}$ ) was added dropwise to a solution of 2-bromobicyclo[2.2.1] hepta-2,5-diene $(\mathbf{5}, 0.50 \mathrm{~g}, 2.9 \mathrm{mmol})$ in dry THF (5 $\mathrm{mL})$ at $-78{ }^{\circ} \mathrm{C}$ and the resulting mixture was stirred for $1 \mathrm{~h}$. Trimethyltin chloride $(582 \mathrm{mg}, 2.9 \mathrm{mmol})$ was added portionwise and then left to warm to room temperature. The mixture was stirred over night at room temperature. The crude product was washed with water $(50 \mathrm{~mL})$ and extracted with $\mathrm{Et}_{2} \mathrm{O}(2 \times$ $50 \mathrm{~mL}$ ) and then the combined ethereal extracts were dried over $\mathrm{MgSO}_{4}$ and concentrated in vacuo. (Bicyclo[2.2.1] hepta-2,5dien-2-yl)trimethylstannane (15) was obtained in the form of a yellow liquid (700 mg, 95\%). ${ }^{1} \mathrm{H} \mathrm{NMR}\left(400 \mathrm{MHz}, \mathrm{CDCl}_{3}\right): \delta$ $7.02\left(\mathrm{bd}, J_{3,4}=2.9 \mathrm{~Hz}, 1 \mathrm{H}, \mathrm{H}^{3}\right), 6.70\left(\mathrm{~m}, 1 \mathrm{H}, \mathrm{H}^{5}\right.$ or $\left.\mathrm{H}^{6}\right), 6.65$ $\left(\mathrm{m}, 1 \mathrm{H}, \mathrm{H}^{5}\right.$ or $\left.\mathrm{H}^{6}\right), 3.76\left(\mathrm{~m}, 1 \mathrm{H}, \mathrm{H}^{1}\right.$ or $\left.\mathrm{H}^{4}\right) 3.63\left(\mathrm{~m}, 1 \mathrm{H}, \mathrm{H}^{1}\right.$ or $\left.\mathrm{H}^{4}\right), 1.91\left(\mathrm{~m}, 1 \mathrm{H}, \mathrm{H}^{7 s y n}\right.$ or $\left.\mathrm{H}^{7 a n t i}\right), 1.88\left(\mathrm{~m}, 1 \mathrm{H}, \mathrm{H}^{7 s y n}\right.$ or $\left.\mathrm{H}^{7 \text { anti }}\right), 0.12\left(\mathrm{~s}, 9 \mathrm{H}, 3 \times \mathrm{CH}_{3}\right) \cdot{ }^{13} \mathrm{C} \mathrm{NMR}\left(100 \mathrm{MHz}, \mathrm{CDCl}_{3}\right): \delta$ 155.46, 154.28, 143.12, 143.07, 74.70, 55.77, 52.15, -9.90.

\section{Reaction of (bicyclo[2.2.1]hepta-2,5-dien-2-yl)trimethylstan-} nane (15) with $\mathbf{C u}\left(\mathrm{NO}_{3}\right)_{2} \cdot \mathbf{3 H}_{2} \mathrm{O}$ : Copper(II) nitrate trihydrate $(1.13 \mathrm{~g}, 4.69 \mathrm{mmol})$ was added portionwise to a solution of $\mathbf{1 5}$ $(1.2 \mathrm{~g}, 4.69 \mathrm{mmol})$ in THF $(10 \mathrm{~mL})$ at room temperature. The blue solution turned green within $40 \mathrm{~min}$. The crude reaction mixture was diluted with $\mathrm{Et}_{2} \mathrm{O}(100 \mathrm{~mL})$ and then washed with $5 \% \mathrm{NH}_{3}(15 \mathrm{~mL})$. The organic phase was dried over $\mathrm{MgSO}_{4}$ and concentrated in vacuo. The syn-dimer 16 and anti-dimer 17 (in a $46: 54$ ratio) were obtained as a mixture $(130 \mathrm{mg}, 30 \%)$. The isomeric dimers 16 [30] and 17 [30] could not be separated and were used as the mixture for the following step.

\section{Cycloaddition reaction of syn-16 and anti-17 mixture with} PTAD: A solution of mixture of syn-16 and anti-17 (120 mg, $0,66 \mathrm{mmol})$ and PTAD (116 mg, 0,66 mmol) in $10 \mathrm{~mL}$ of $\mathrm{CH}_{2} \mathrm{Cl}_{2}$ was stirred at room temperature for $30 \mathrm{~min}$. The solvent was removed under reduced pressure. The residue was chromatographed on silica gel (30 g) column eluted with EtOAc/nhexane (1:9).

The first fraction was anti-cycloadduct $20(89 \mathrm{mg}, 70 \%$ based on anti dimer 17). Yellowish crystals from $\mathrm{CH}_{2} \mathrm{Cl}_{2} / n$-hexane
(2:1), mp: $174-176{ }^{\circ} \mathrm{C} .{ }^{1} \mathrm{H}$ NMR $\left(400 \mathrm{MHz}, \mathrm{CDCl}_{3}\right): \delta$ 7.53-7.25 (m, 5H, H), 6.33-6.27 (m, 3H), 6.06 (dd, $J=5.5 \mathrm{~Hz}$, $J=2.9 \mathrm{~Hz}, 1 \mathrm{H}), 4.33(\mathrm{~d}, J=3.6 \mathrm{~Hz}, 1 \mathrm{H}), 4.09(\mathrm{~m}, 1 \mathrm{H}), 3.93$ $(\mathrm{m}, 1 \mathrm{H}), 3.68(\mathrm{~d}, J=1.7,1 \mathrm{H}), 3.54(\mathrm{~m}, 2 \mathrm{H}), 1.88(\mathrm{dt}$, A part of AB system, $J=9.2 \mathrm{~Hz}, J=1.7 \mathrm{~Hz}, 1 \mathrm{H}$ ), 1.77 (bd, A part of AB system, $J=8.8 \mathrm{~Hz}, 1 \mathrm{H}$ ), 1.68 (bd, B part of AB system, $J=9.2$ $\mathrm{Hz}, 1 \mathrm{H}), 1.54$ (bd, B part of AB system, $J=8.8 \mathrm{~Hz}, 1 \mathrm{H}) .{ }^{13} \mathrm{C}$ NMR (100 MHz, $\left.\mathrm{CDCl}_{3}\right): \delta 151.07,149.85,136.64,136.55$, 135.80, 133.12, 131.90, 131.61, 131.34, 129.20, 128.05, 125.87, 58.26, 56.54, 48.04, 47.57, 46.72, 46.37, 45.59, 45.38. IR (KBr, $\left.\mathrm{cm}^{-1}\right): 3060,2925,2852,1760,1698,1502,1419,1130,1028$, 721. MS (70 eV) m/z: $357.3\left(\mathrm{M}^{+}, 19\right), 315.8$ (16), 291.2 (93), 250.9 (21), 239.2 (41), 195.1 (22), 182.1 (82), 118.8 (90), 91.0 (77), 77.0 (44). The second fraction was syn-cycloadduct 18 (75 $\mathrm{mg}, 69 \%$ based on syn dimer 16) Yellowish crystals from $\mathrm{CH}_{2} \mathrm{Cl}_{2} / n$-hexane (2:1), mp: $194-196{ }^{\circ} \mathrm{C} .{ }^{1} \mathrm{H} \mathrm{NMR}(400 \mathrm{MHz}$, $\left.\mathrm{CDCl}_{3}\right): \delta 7.54-7.34\left(\mathrm{~m}, 5 \mathrm{H}, \mathrm{H}_{\text {aryl }}\right), 6.28(\mathrm{~m}, 4 \mathrm{H}), 4.10(\mathrm{~m} 2 \mathrm{H})$, $3.73(\mathrm{~m}, 2 \mathrm{H}), 3.64(\mathrm{~m}, 2 \mathrm{H}), 1.84$ (bd, A Part of AB system, $J=$ $9.0 \mathrm{~Hz}, 2 \mathrm{H}), 1.69$ (bd, B part of AB system, $J=9.0 \mathrm{~Hz}, 2 \mathrm{H})$. ${ }^{13} \mathrm{C} \mathrm{NMR}\left(100 \mathrm{MHz}, \mathrm{CDCl}_{3}\right): \delta 151.47,136.83,135.82$, $131.83,131.05,129.25,128.19,126.06,57.94,48.10,46.20$, 45.66. IR (KBr, cm $\left.{ }^{-1}\right): 3060,2962,2929,2863,1760,1700$, 1502, 1422, 1279, 1139, 761, 729. MS (70 eV) m/z: $357.4\left(\mathrm{M}^{+}\right.$, 4), 316.4 (4), 291.3 (54), 280.1 (3), 252.0 (4), 239.0 (7), 210.3 (9), 182.1 (25), 165.1 (35), 144.0 (71), 120.0 (16), 115.0 (40), $102.0(9), 90.7$ (65), 66.1 (23).

Cycloaddition reaction of syn-16 and anti-17 mixture with TCNE (10): A solution of mixture of syn-16 and anti-17 (103 $\mathrm{mg}, 0.56 \mathrm{mmol})$ and TCNE (10, $72 \mathrm{mg}, 0.56 \mathrm{mmol})$ in $10 \mathrm{~mL}$ of $\mathrm{CH}_{2} \mathrm{Cl}_{2}$ was stirred at room temperature overnight. The solvent was removed under reduced pressure. The residue was chromatographed on silica gel (30 g) eluted with EtOAc/ $n$-hexane (1:32). The first fraction was anti-1,4,5,8,8a,10a-hexahydro1,4:5,8-dimethanophenanthrene-9,9,10,10-tetracarbonitrile (21) (82 mg, 87\% based on anti dimer 17). Yellowish crystals from $\mathrm{CH}_{2} \mathrm{Cl}_{2} / n$-hexane $(3: 1), \mathrm{mp}: 160-162{ }^{\circ} \mathrm{C} .{ }^{1} \mathrm{H} \mathrm{NMR}(400 \mathrm{MHz}$, $\left.\mathrm{CDCl}_{3}\right): \delta 6.46-6.35(\mathrm{~m}, 4 \mathrm{H}), 3.61(\mathrm{~m}, 2 \mathrm{H}), 3.49(\mathrm{~m}, 1 \mathrm{H})$, 3.36-3.33 (m, 2H), $2.36(\mathrm{~m}, 1 \mathrm{H}), 2.16(\mathrm{~d}$, A part of AB system, $J=9.9 \mathrm{~Hz}, 1 \mathrm{H}), 1.95$ (d, B Part of AB system, $J=9.9 \mathrm{~Hz}, 1 \mathrm{H})$, $1.74-1.71(\mathrm{~m}, 2 \mathrm{H}) .{ }^{13} \mathrm{C} \mathrm{NMR}\left(100 \mathrm{MHz}, \mathrm{CDCl}_{3}\right): \delta 138.63$, 138.37, 138.27, 132.47, 131.96, 130.76, 113.25, 112.03, 110.50, $110.47,51.40,51.32,48.01,46.74,46.47,46.43,45.63,45.62$, 44.79, 44.75. IR (KBr, cm $\left.{ }^{-1}\right): 2934,2896,2868,2352,2093$, 1457, 1235, 1043, 960. MS (70 eV) m/z: $310.1\left(\mathrm{M}^{+}, 83\right), 295.0$ (17), 282.1 (70), 268.0 (57), 243.0 (73), 229.0 (55), 218.1 (80), 203.0 (48), 190.0 (52), 179.0 (40), 167.0 (100), 151.9 (53). The second fraction was syn-1,4,5,8,8a,10a-hexahydro-1,4:5,8dimethanophenanthrene-9,9,10,10-tetracarbonitrile (19) $(75 \mathrm{mg}$, $93 \%$ based on syn dimer 16). Colorless crystals from $\mathrm{CH}_{2} \mathrm{Cl}_{2} / n$ hexane (2:1), mp: $136-138{ }^{\circ} \mathrm{C} .{ }^{1} \mathrm{H}$ NMR (400 MHz, $\left.\mathrm{CDCl}_{3}\right): \delta$ 
$6.43(\mathrm{dd}, J=5.5 \mathrm{~Hz}, J=3.3 \mathrm{~Hz}, 2 \mathrm{H}), 6.30(\mathrm{dd}, J=5,5 \mathrm{~Hz}, J=$ $2.9 \mathrm{~Hz}, 2 \mathrm{H}), 3.66$ (s, 2H), $3.36(\mathrm{~d}, J=1.3 \mathrm{~Hz}, 2 \mathrm{H}), 2.48$ (d, $J=$ $1.3 \mathrm{~Hz}, 2 \mathrm{H}), 2.10$ (d, A Part of AB system, $\left.J_{i}=9.5 \mathrm{~Hz}, 2 \mathrm{H}\right)$, 1.89 (d, B Part of AB system, $J=9.5 \mathrm{~Hz}, 2 \mathrm{H}) .{ }^{13} \mathrm{C}$ NMR (100 $\mathrm{MHz}, \mathrm{CDCl}_{3}$ ): $\delta 138.25,137.78,132.26,112.39,110.79,49.49$, 47.90, 46.52, 45.95, 45.30. IR (KBr, $\left.\mathrm{cm}^{-1}\right): 3066,2995,2951$, $2874,2247,1454,1317,1007,727$. MS (70 eV) $\mathrm{m} / \mathrm{z}: 310.1$ $\left(\mathrm{M}^{+}, 35\right), 282.1$ (24), 268.1 (25), 242.1 (27), 228.1 (25), 217.1 (35), 204.1 (18), 189.0 (24), 178.1 (19), 167.1 (38), 126.9 (20), 115.2 (30), 101.3 (14), 91.0 (18), 88.1 (22), 76.1 (18), 65.5 (100).

\section{Acknowledgments}

This work has been supported by TUBITAK (project no 106T082), TUBA (Turkish Academy of Sciences, under the Young Scientist Award Program (AD/TUBA-GEBIP/2001-13)) and Atatürk University. We are indebted for their financial support.

\section{References}

1. Gassman, P. G.; Gennick, I. J. Org. Chem. 1980, 45, 5211-5213. doi:10.1021/jo01313a039

2. Horasan (Kishali), N.; Kara, Y.; Azizoğlu, A.; Balci, M. Tetrahedron 2003, 59, 3691-3699. doi:10.1016/S0040-4020(03)00549-0

3. Tutar, A.; Taşkesenligil, Y.; Çakmak, O.; Abbasoğlu, R.; Balci, M. J. Org. Chem. 1996, 61, 8297-8300. doi:10.1021/jo960225I

4. Winstein, S. J. Am. Chem. Soc. 1961, 83, 1516-1517. doi:10.1021/ja01467a058

5. Dastan, A. Tetrahedron 2001, 57, 8725-8732. doi:10.1016/S0040-4020(01)00851-1

6. Daştan, A.; Demir, U.; Balci, M. J. Org. Chem. 1994, 59, 6534-6538. doi:10.1021/jo00101a011

7. Cristol, S. J.; Nachtigall, G. W. J. Org. Chem. 1967, 32, 3727-3737. doi:10.1021/jo01287a001

8. Wilt, J. W.; Chenier, P. J. J. Org. Chem. 1970, 35, 1562-1570. doi:10.1021/j000830a065

9. Daştan, A.; Balci, M. Tetrahedron 2005, 61, 5481-5488. doi:10.1016/j.tet.2005.03.132

10. Gültekin, D. D.; Taşkesenligil, Y.; Daştan, A.; Balcı, M. Tetrahedron 2008, 64, 4377-4383. doi:10.1016/j.tet.2008.02.067

11. Barkhash, V. A. Top. Curr. Chem. 1984, 116-117, 1-265. doi:10.1007/3-540-12555-8_1

12. Zimmerman, H. E. In Rearrangements in Ground and Excited States; de Mayo, P., Ed.; Academic Press: New York, 1980; Vol. 3, Essay 16, pp 131-164.

13. Hemetsberger, H.; Nispel, F. Tetrahedron 1990, 46, 3823-3840. doi:10.1016/S0040-4020(01)90518-6

14. Johnson, R. P.; Schlimgen Davis, D. Tetrahedron Lett. 1981, 22, 3171-3174. doi:10.1016/S0040-4039(01)81855-4

15. Altundas, R.; Dastan, A.; Ünaldi, N. S.; Güven, K.; Uzun, O.; Balci, M. Eur. J. Org. Chem. 2002, 526-533.

doi:10.1002/1099-0690(20022)2002:3<526::AID-EJOC526>3.0.CO;2-N

16. Tang, H.; Dong, Z.; Merican, Z.; Margetić, D.; Marinić, Ž.; Gunter, M. J.; Officer, D.; Butler, D. N.; Warrener, R. N. Tetrahedron Lett. 2009, 50, 667-670. doi:10.1016/j.tetlet.2008.11.109
17. Lledó, A.; Solà, J.; Verdaguer, X.; Riera, A.; Maestro, M. A. Adv. Synth. Catal. 2007, 349, 2121-2128. doi:10.1002/adsc. 200700145

18. Altuna-Urquijo, M.; Gehre, A.; Stanforth, S. P.; Tarbit, B. Tetrahedron 2009, 65, 975-984. doi:10.1016/j.tet.2008.11.090

19. Tseng, N.-W.; Lautens, M. J. Org. Chem. 2009, 74, 1809-1811. doi:10.1021/j0802622d

20. Zhou, J. (S.); Hartwig, J. F. J. Am. Chem. Soc. 2008, 130, 12220-12221. doi:10.1021/ja803523z

21. Rendler, S.; Froehlich, R.; Keller, M.; Oestreich, M. Eur. J. Org. Chem. 2008, 2582-2591. doi:10.1002/ejoc.200800107

22. Dalkılıç, E.; Güney, M.; Daştan, A.; Saracoglu, N.; De Lucchi, O.; Fabris, F. Tetrahedron Lett. 2009, 50, 1989-1991. doi:10.1016/j.tetlet.2009.02.070

23. Zonta, C.; Cossu, S.; De Lucchi, O. Eur. J. Org. Chem. 2000, 1965-1971.

doi:10.1002/(SICI)1099-0690(200005)2000:10<1965::AID-EJOC1965> 3.0.CO;2-C

24. Borsato, G.; Linden, A.; De Lucchi, O.; Lucchini, V.; Wolstenholme, D.; Zambon, A. J. Org. Chem. 2007, 72, 4272-4275. doi:10.1021/jo070222g

25. Peluso, P.; Greco, C.; De Lucchi, O.; Cossu, S. Eur. J. Org. Chem. 2002, 4024-4031. doi:10.1002/1099-0690(200212)2002:23<4024::AID-EJOC4024>3.0.C $\mathrm{O} ; 2-\mathrm{N}$

26. Tranmer, G. K.; Yip, C.; Handerson, S.; Jordan, R. W.; Tam, W. Can. J. Chem. 2000, 78, 527-535. doi:10.1139/cjc-78-5-527

27. Kenndoff, J.; Polborn, K.; Szeimies, G. J. Am. Chem. Soc. 1990, 112, 6117-6118. doi:10.1021/ja00172a031

28. Stille, J. K. Angew. Chem., Int. Ed. Engl. 1986, 25, 508-524. doi:10.1002/anie.198605081

29. Fabris, F.; Leoni, L.; De Lucchi, O. Tetrahedron Lett. 1999, 40 , 1223-1226. doi:10.1016/S0040-4039(98)02571-4

30. Baumgärtel, O.; Szeimies, G. Chem. Ber. 1983, 116, 2180-2204. doi:10.1002/cber.19831160612

\section{License and Terms}

This is an Open Access article under the terms of the Creative Commons Attribution License (http://creativecommons.org/licenses/by/2.0), which permits unrestricted use, distribution, and reproduction in any medium, provided the original work is properly cited.

The license is subject to the Beilstein Journal of Organic Chemistry terms and conditions:

(http://www.beilstein-journals.org/bjoc)

The definitive version of this article is the electronic one which can be found at: doi:10.3762/bjoc.5.39 